\title{
Nonlinear optical characterization of the gold nanoparticles coated by thiols
}

\author{
Piotr Lesiak ${ }^{* 1}$ and Michał Wójcik ${ }^{2}$ \\ ${ }^{1}$ Faculty of Physics, Warsaw University of Technology, Koszykowa 75, 00-662 Warszawa, \\ ${ }^{2}$ Department of Chemistry, University of Warsaw, Al. Zwirki i Wigury 101, 02-089 Warszawa
}

Received September 13, 2011; accepted September 29, 2011; published September 30, 2011

\begin{abstract}
Structured nanomaterials have great potential applications based on their unusual optical properties, such as a negative refractive index [1], plasmon waveguiding [2] or nonlinear optics. In this paper we present the nonlinear optical characterization of gold nanoparticles coated by thiols with the ratio of mesogenic to n-alkyl thiols varying from 1:2 to $1: 1$ in a toluene suspension using the Z-scan technique. The type of mesogenic molecule in the metal coating had a profound influence on the gold-cluster self-assembly. To check how these clusters affect the nonlinear properties we use two different polarizations of a laser beam - linear and circular.
\end{abstract}

Nonlinear optical materials are of great practical importance for future applications in optical signal processing, optical limiting and nonlinear optical devices. Nanoparticle suspensions in dielectric solvents have been widely investigated due to their large third-order susceptibility and ultra-fast response time. Nanoparticles of alkali metals and noble metals copper, silver and gold show broad absorption bands in the visible region of the electromagnetic spectrum. The plasmon absorption band and the optical response can be changed by altering nanoparticle size and shape. The interaction of light with metal nanoparticles has received a large amount of attention in the past decade.

In this paper the nonlinear optical characterization of a nanoparticle suspension was performed using the Z-scan technique. In this technique a polarized Gaussian laser beam, propagating in the $z$-direction, is focused to a narrow waist [3-5]. The sample is moved along the $\mathrm{z}$ direction and the transmitted intensity is measured through a finite aperture in the far field as a function of the sample position $\mathrm{z}$; measured with respect to the focal plane. As the sample moves through the beam focus (at $z=0$ ), selffocusing or -defocusing modifies the wave front phase, thereby modifying detected beam intensity. The nonlinear coefficient of refraction and absorption can be measured with a close and open aperture, respectively.

The phase imposed by the nonlinear refraction of the sample appears as spatial beam broadening or narrowing in the far field. These beam shape changes modify the fraction of light that passes through a small aperture as the

\footnotetext{
*E-mail: lesiak@if.pw.edu.pl
}

sample is moved along the beam propagation direction. For a Gaussian beam with waist $\omega_{0}$, and diffraction length $z_{0}=\pi \omega_{0}^{2} / \lambda$ the transmittance of the sample of thickness $L$ with a fully open aperture gives a measure of nonlinear absorption, and is given approximately by:

$$
T(x)=1-\frac{1}{2 \sqrt{2}\left(1+x^{2}\right)} \Delta \Psi,
$$

where $x=z / z_{0}$ and $\Delta \Psi$ is the imaginary nonlinear phase shift. The transmittance with a closed aperture gives a measure of nonlinear diffraction together with nonlinear absorption and given approximately by:

$$
T(x)=1-\frac{4 x}{\left(1+x^{2}\right)\left(9+x^{2}\right)} \Delta \Phi-\frac{1}{2 \sqrt{2}\left(1+x^{2}\right)} \Delta \Psi,
$$

where $\Delta \Phi=k \gamma I_{0}\left(1-e^{-\alpha_{0} L}\right) / \alpha_{0}$ is the nonlinear phase shift. From the transmittance curve, the value of the medium nonlinear refractive index $\mathrm{n}_{2}$ are obtained using the expression

$$
n_{2}=c \varepsilon_{0} n_{0} k \frac{\Delta T_{p-v}}{0.406 I_{0} L_{e f f}(1-A)^{0.25}}
$$

where: $L_{\text {eff }}=\left(1-e^{-\alpha_{0} L}\right) / \alpha_{0}$ is the effective thickness of the sample; $L$ is the original thickness of the nanoparticle suspension; $k$ is the wave vector; $A=d_{\text {close }} / d_{\text {open }} ; d$ is the close and open aperture diameter; $\Delta T_{p-v}$ is the peak-valley distance in the close aperture z-scan curve.

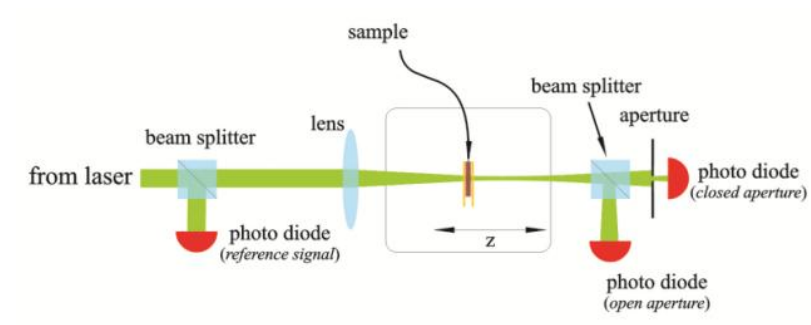

Fig. 1. Z-scan setup.

A scheme of the setup of the z-scan technique is shown in Fig. 1. As a CW light source an He-Ne green (543nm) and 
red $(633 \mathrm{~nm})$ laser has been used. In both cases, the beam diameter was $0.9 \mathrm{~cm}$ and the beam spot radius at the focal point measured 7 microns. Different wavelengths applied allow to measure nonlinear refractive index close and far from the collective plasma oscillations of the free electrons gas of the gold nanoparticles.

The cuvette is fixed on a microprocessor controlled translation stage that has a range of $10 \mathrm{~cm}$ and a resolution of 2.5 microns, so that it can be accurately moved through the focal region of the laser beam. Data acquisition is facilitated in real time by the use of a PC.
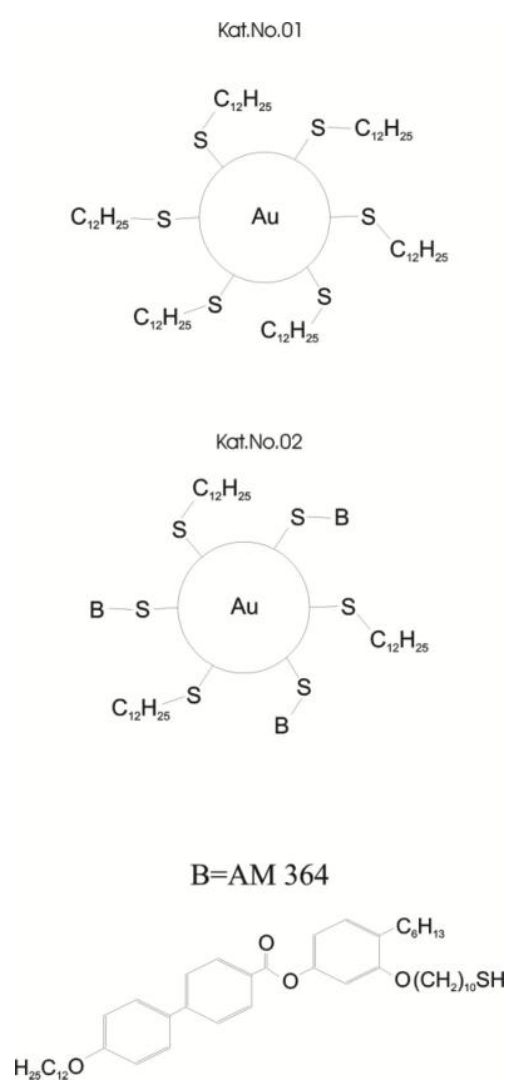

Fig. 2. Molecular structures of thiols and mesogenic thiols.

The size of the metal center, $2 \mathrm{~nm}$ with a distribution of $0.2 \mathrm{~nm}$ for both samples studied, was determined by direct TEM imaging, by X-ray scattering of a diluted NP solution. We deduced from NMR spectroscopic and elementary analyses that approximately $65 \%$ of the metal surface was coated by thiols (Kat.No.01). For the second sample (Kat.No.02) the metal surface was coated by thiols with the ratio of mesogenic to n-alkyl thiols varying from $1: 2$ to $1: 1$, depending on the reaction time for the exchange (Fig. 2.). This result indicates that between 25 and 40 mesogenic groups are attached to a single gold particle. The type of mesogenic molecule in the metal coating had a profound influence on gold-cluster selfassembly. To check how these clusters affect the nonlinear properties we use two different polarizations of the laser beam - linear and circular.

Figure 3 shows absorption spectra for both nanoparticle types of suspension with resonances around 510nm, caused by collective plasma oscillations of free electron gas, and typical close aperture z-scan results for nanoparticle suspension are shown in Fig. 4.

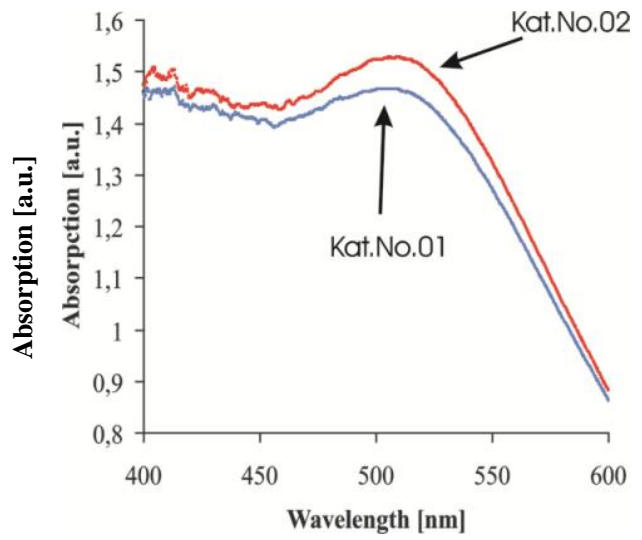

Fig. 3. Absorption spectrum for both nanoparticle types.

Measurements made for two polarizations of the laser beam (linear and circular) indicate that for the circular polarization the nonlinear refractive index is smaller than for the linear one.

Tab. 1. Comparison between nonlinear phase shifts calculated from measurements for linear and circular polarizations.

\begin{tabular}{|c|c|c|}
\hline \multicolumn{1}{|c|}{} & Kat.No.01 & Kat. No.02 \\
\hline Beam waist & \multicolumn{2}{|c|}{$7 \mu \mathrm{m}$} \\
\hline Power & \multicolumn{2}{|c|}{$0.4 \mu \mathrm{J}$} \\
\hline $\mathbf{A}$ & \multicolumn{2}{|c|}{0.5} \\
\hline $\mathbf{n}_{\mathbf{2}}\left[\mathbf{m}^{\mathbf{2}} / \mathbf{W}\right]$ (linear) & $1.57 \cdot 10^{-14}$ & $2.62 \cdot 10^{-14}$ \\
\hline $\mathbf{n}_{\mathbf{2}}\left[\mathbf{m}^{\mathbf{2}} / \mathbf{W}\right]$ (circ.) & $1.29 \cdot 10^{-14}$ & $0.72 \cdot 10^{-14}$ \\
\hline
\end{tabular}

The canonical picture of photophysics of a gold nanoparticle, surfactant and solvent system is that light excites electrons, which subsequently relax by electronelectron (e-e) scattering to give a hot electron distribution. The hot electrons then equilibrate with the lattice by electron-phonon (e-ph) coupling. This sequence of events is identical to what happens in bulk metals. In bulk metals, the energy deposited by the laser diffuses away from the excitation region in a way that depends on the thermal conductivity of the metal. In contrast, for particles, the final step in the relaxation process is the transfer of energy from the hot electron/phonon system to the environment. 
Although light absorption is a local process, the diffusion of heat gives rise to a nonlinear optical phenomenon which has a nonlocal nature. The hotter region is located in the center of the beam $(r=0, r$ being the radial distance from the beam axis). The index of refraction $n$ of the medium, as a function of $r$ and the time $t$, can be written as:

$$
n(r, t)=n_{0}+\frac{\partial n}{\partial T} \Delta T(r, t),
$$

where $\partial n / \partial T$ is the thermo-optic coefficient for pure toluene and $\Delta T$, between two positions $x_{1}$ and $x_{2}$ at the centre of the beam and far from the heated region respectively, can be written as:

$$
\Delta T=\frac{\alpha_{0} I_{0} \ln \left(x_{2} / x_{1}\right)}{2 \pi \kappa L},
$$

where $\lambda$ and $I_{0}$ are the wavelength and incident light intensity, respectively, and $\kappa$ is the thermal conductivity.
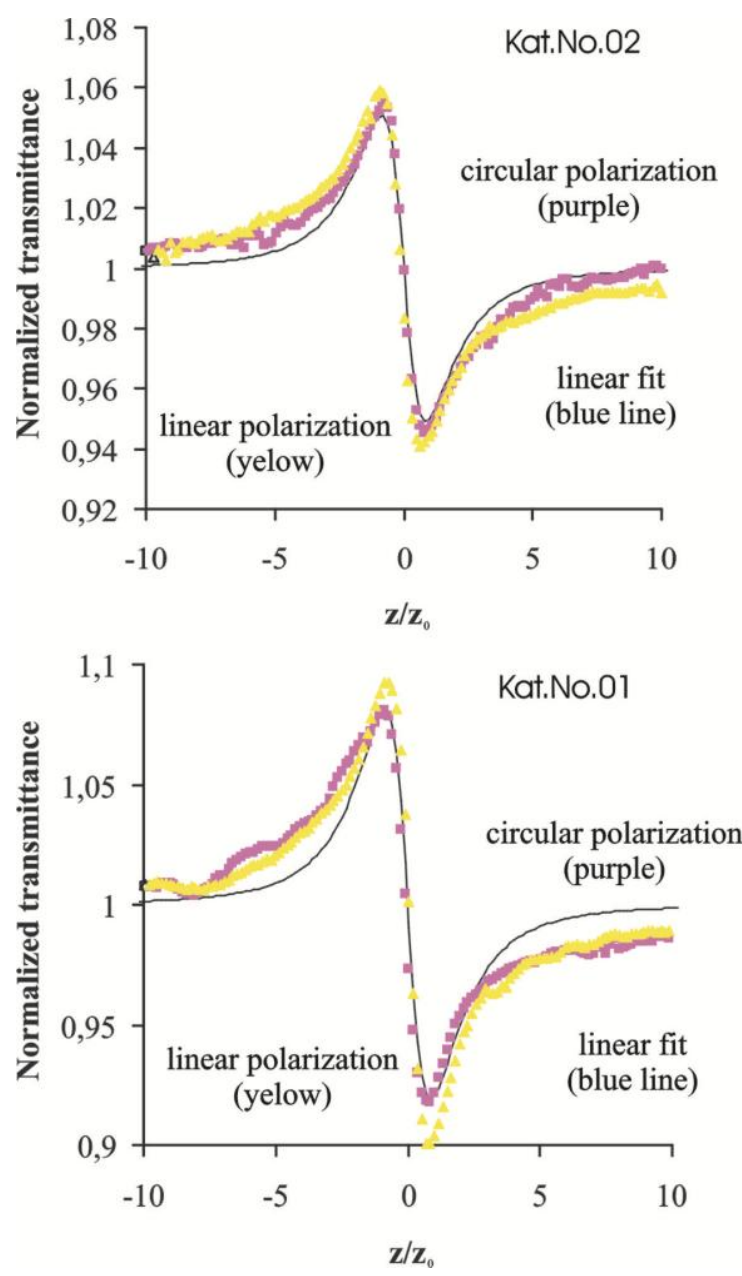

Fig. 4. Nonlinear refractive index measurements made for $543 \mathrm{~nm}$ (up) and $633 \mathrm{~nm}$ (bottom).
In our experiment the heating temperature was $0.1 \mathrm{~K}$. The relaxation time at position $\mathrm{z}$ is given by:

$$
t_{c}(z)=\frac{\omega_{z}^{2}}{4 D},
$$

where $\omega=\left[\omega_{0}^{2}\left(1+x^{2}\right)\right]^{1 / 2}$ is the radius of the beam at position $z$, and $D$ is the thermal diffusivity of the pure toluene. In our experiment the relaxation time was equal to $150 \mathrm{~ms}$. If we use Eq. (5), the nonlinear phase shift for known temperature difference in the hotter region can be written as:

$$
\Delta \varphi=\frac{\alpha_{0} I}{\lambda \kappa} \frac{\partial n}{\partial T} .
$$

Simultaneously, the nonlinear phase shift can be calculated from the nonlinear refractive index:

$$
\Delta \varphi=\frac{n_{2} L \pi}{\lambda}
$$

Tab. 2. Comparison between nonlinear phase shifts calculated from measurements and from the heating process for two different nanoparticle suspensions (linear polarizaton case) at $633 \mathrm{~nm}$.

\begin{tabular}{|c|c|c|}
\hline \multicolumn{1}{|c|}{} & Kat.No.01 & Kat.No.02 \\
\hline $\mathbf{n}_{\mathbf{2}}\left[\mathbf{m}^{\mathbf{2}} / \mathbf{W}\right]$ & $0.15 \cdot 10^{-13}$ & $0.26 \cdot 10^{-13}$ \\
\hline$\Delta \boldsymbol{\varphi}(\mathbf{E q}$. (8)) & 0.78 & 1.58 \\
\hline$\Delta \mathbf{T}[\mathbf{K}]$ & 0.12 & 0.23 \\
\hline$\Delta \boldsymbol{\varphi}$ (Eq. (7)) & 0.85 & 1.43 \\
\hline
\end{tabular}

As presented in Tab. 2, the nonlinear phase shifts calculated in two different ways are similar.

In conclusion, as shown in this paper, the nonlinear optical properties of gold nanoparticles coated by thiols with the ratio of mesogenic to n-alkyl thiols varying from 1:2 to $1: 1$ in a toluene suspension depends on the polarization of the laser beam. For circular polarization the nonlinear refractive index is smaller than for linear polarization.

P.L. would like to acknowledge the Foundation for Polish Science (Mistrz Programme).

\section{References}

[1] J.B. Pendry, Phys. Rev. Lett. 85, 3966 (2000).

[2] S.A. Maier, M.L. Brongersma, P.G. Kik, S. Meltzer, A.A.G. Requicha, H.A. Atwater, Adv. Mater. 13, 1501 (2001).

[3] M. Sheik-Bahae, A.A. Said, E.W. Van Stryland, Opt. Lett. 14, 955 (1989).

[4] M. Sheik-Bahae, A.A. Said, T.H. Wei, D.J. Hagan, E.W. Van Stryland, J. Quant. Electr. 26, 760 (1990).

[5] B.E.A. Saleh, M.C. Teich, Fundamentals of Photonics (John Wiley \& Sons, Inc., second edition, 2007). 